\title{
Preparation and Characterization of the Phthalazinone Structure-based Microporous Organic Polymer
}

\author{
Kuanyu Yuan ${ }^{1, a}$, Cheng Liu ${ }^{1, b_{\star}}$, Hongmin Duan ${ }^{2, c}$, Manxia Zhang ${ }^{3, d}$, Xigao \\ Jian $^{1, e}$ \\ ${ }^{1}$ Department of Polymer Science \& Materials, Dalian University of Technology, Dalian, 116024, \\ China \\ ${ }^{2}$ Dalian Institute of Chemical Physics, Chinese Academy of Sciences, Dalian, 116023, China \\ ${ }^{3}$ Institute of Environmental Pollution and Remediation, Dalian Maritime University, Dalian, 116026, \\ China \\ bemail: liuch1115@dlut.edu.cn
}

Keywords: Phthalazinone; Triazine; Microporous

\begin{abstract}
In this work, a new covalent triazine framework based on phthalazinone-containing dinitrile building blocks was prepared by the dynamic nitrile-trimerization reaction. The FT-IR spectra of BCTF illustrated the characteristic peaks of the triazine at $1503 \mathrm{~cm}^{-1}$ and $1360 \mathrm{~cm}^{-1}$. The nitrogen sorption isotherm (Type-I) showed a rapid uptake at low pressure (0-0.1bar) indicating a permanent microporous nature. Pore size distribution lower than $2 \mathrm{~nm}$ was calculated using nonlocal density functional theory (NL-DFT). TGA results showed that BCTF polymer with the skeleton decomposition temperature above $500^{\circ} \mathrm{C}$ had relatively excellent thermal stability.
\end{abstract}

\section{Introduction}

Microporous organic polymers (MOPs) are porous materials with pores smaller than $2 \mathrm{~nm}$, which are composed of light, non-metallic elements such as C, H, O, N and B [1]. Recently, MOPs have attracted considerable attention and interest in this field of gas storage and selected separation [2], while possessing high thermal and chemical stability, steerable pore size distribution and high micoropore volumes with cost-effective and diversified synthesis procedures [3-4]. On account of numerous available organic monomers and reactions, it is easy to introduce various functional groups in the pore walls and be flexible for the material design to achieve desirable pore properties with a plethora of building blocks. In the past few years, several types MOPs have been successfully manifested, such as hyper-crosslinked polymers (HCPs) [5], polymers of intrinsic microporosity (PIMs) [6], covalent organic frameworks (COFs) [7] and conjugated microporous polymers (CMPs) [8]. Covalent triazine-based frameworks (CTFs) [9] were developed through reversible ionothermal trimerization of cheap and available easily aromatic nitriles catalyzed by a Lewis acid under conditions of high temperature and autogenic pressure [10]. CTFs exhibit large specific surface areas, as well as remarkable thermal stabilities, tunable pore sizes, and high gas adsorption capacities [3-4]. In addition, CTFs have been used in heterogeneous catalysis, as a catalytic support in liquid phase reactions, for gas storage and separation of organic dyes [11].
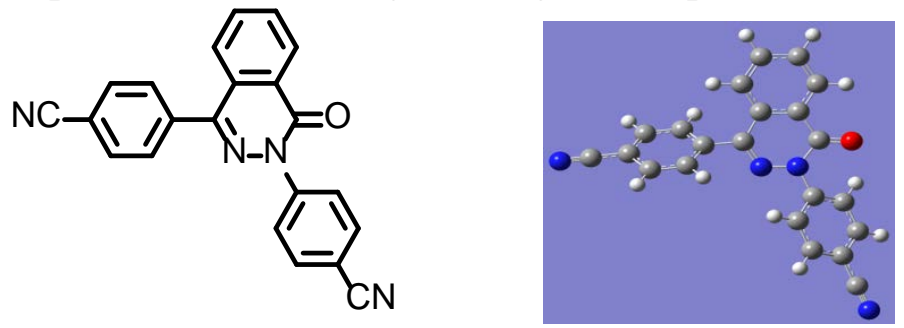

Fig 1. The structure of BHPZ-DN (a) and molecular analog by Gaussian 09 (b).

In this study, we designed and synthesized a novel aromatic dinitrile with twisted phthalazinone moiety 2-(4'- cyanophenyl)- 4-(4'- cyanophenyl) -2, 3-phthalazin-1-one (BHPZ-DN) (Fig 1a). The 
rigid phthalazinone core and the twisted space between the core and the nitrile groups may help to increase the available space for gas adsorption and storage. Using molten $\mathrm{ZnCl}_{2}$ as both molten solvent and catalyst at $400^{\circ} \mathrm{C}$, we obtained a new porous phthalazinone-based covalent triazine-containing organic framework (PHCTF) (Scheme 1). It displays comparatively high thermal stability and high apparent surface area.

\section{Experimental Section}

Materials BHPZ-DN was synthesized by our group. Zinc chloride was refluxed and distilled over thionyl chloride to remove water, and then excess thionyl chloride was azeotropically distilled with toluene. After the removal of thionyl chloride and toluene, anhydrous zinc chloride was obtained after being dried at $180{ }^{\circ} \mathrm{C}$ under vacuum for $24 \mathrm{~h}$.

Characterization Methods FT-IR spectrum was performed on a Thermo Nicolet Nexus 470 Fourier transform infrared spectrometer in the $400-4000 \mathrm{~cm}^{-1}$ region ( $\mathrm{KBr}$ pressed disk). Termogravimetry measurement (TG) was performed in the nitrogen atmosphere on a TA Instrument thermal gravimetric analyzer (TGA Q500) at a heating rate of $20{ }^{\circ} \mathrm{C} \mathrm{min}{ }^{-1}$. The dry state polymer surface areas and pore size distributions were performed at an Autosorb iQ (Quantachrome) analyzer. Prior to measurements, the sample was degassed at $150{ }^{\circ} \mathrm{C}$ under high vaccum for $12 \mathrm{~h}$.

Synthesis of PHCTF PHCTF was synthesized by heating a mixture of the synthesized BHPZ-DN $(0.16 \mathrm{~g}, 0.45 \mathrm{mmol})$ and $\mathrm{ZnCl}_{2}(0.61 \mathrm{~g}, 4.5 \mathrm{mmol})$ in a quartz tube $(3 \times 5 \mathrm{~cm})$ according to the literature [12]. The tube was evacuated to a high vacuum and then sealed rapidly. Following by a temperature program $\left(250^{\circ} \mathrm{C}\right.$ for $10 \mathrm{~h}, 300^{\circ} \mathrm{C}$ for $10 \mathrm{~h}, 350^{\circ} \mathrm{C}$ for $10 \mathrm{~h}$, and $400^{\circ} \mathrm{C}$ for $20 \mathrm{~h}$ ), the quartz tube was cooled to room temperature, and the reaction mixture was subsequently ground and then washed thoroughly with water for $72 \mathrm{~h}$ to remove most of the catalyst. After that, the product was isolated by filtration and again stirred with $100 \mathrm{ml} 2 \mathrm{~mol} / \mathrm{L} \mathrm{HCl}$ for $24 \mathrm{~h}$ to remove the residual salt. The resulting black powder was filtered and washed successively with water and alcohol, followed by an overnight Soxhlet extraction using acetone, methyl alcohol as eluting solvent sequentially, and finally dried in vacuum at $150^{\circ} \mathrm{C}$. Yield: $80 \%$.

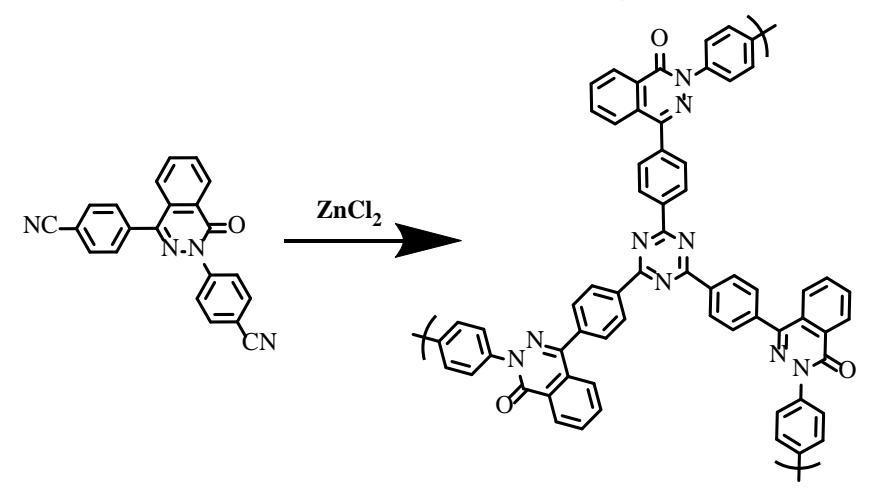

Scheme 1. Synthesis of PHCTF

\section{Results and discussion}

Porous CTF materials can be synthesized by an ionothermal reaction between aromatic nitriles in $\mathrm{ZnCl}_{2}$, which can be adapted to a large scale. Molten $\mathrm{ZnCl}_{2}$ acts as a Lewis acid catalyst, molten solvent and porogen for the polymerization_ENREF_14 [11]. The high-temperature trimerization of aromatic nitrile groups catalyzed by $\mathrm{ZnCl}_{2}$ would form a $\mathrm{C}_{3} \mathrm{~N}_{3}$ triazine ring, which can be marked as a triangular ring. The material PHCTF is insoluble in common organic solvents indicating its good chemical stability. The formation of polytriazine framework was confirmed by Fourier transform infrared (FT-IR) studies (Fig 2 Left). The peak at $1692 \mathrm{~cm}^{-1}$ can be ascribed to the lactam $(\mathrm{C}=\mathrm{O})$ of phthalazinone moiety. The almost disappearance in the intense $\mathrm{C} \equiv \mathrm{N}$ band around 2238 $\mathrm{cm}^{-1}$ was indicative of a high conversion of nitriles after reaction, and formed new characteristic C-N stretching bands for triazine units at $1352 \mathrm{~cm}^{-1}$ and $1497 \mathrm{~cm}^{-1}$ for PHCTF. Concerning the thermal stability of the obtained framework, from thermogravimetric analysis (TGA) (Fig 2 Right), 
it can be observed that the polymer skeleton decomposition started at above $500^{\circ} \mathrm{C}$. Specially, the apparent weight loss before $300^{\circ} \mathrm{C}$ should be attributed to the adsorbed gas, solvent and remaining hydrated water. However, the former TGA analysis still displayed comparatively high thermal stability of PHCTF.
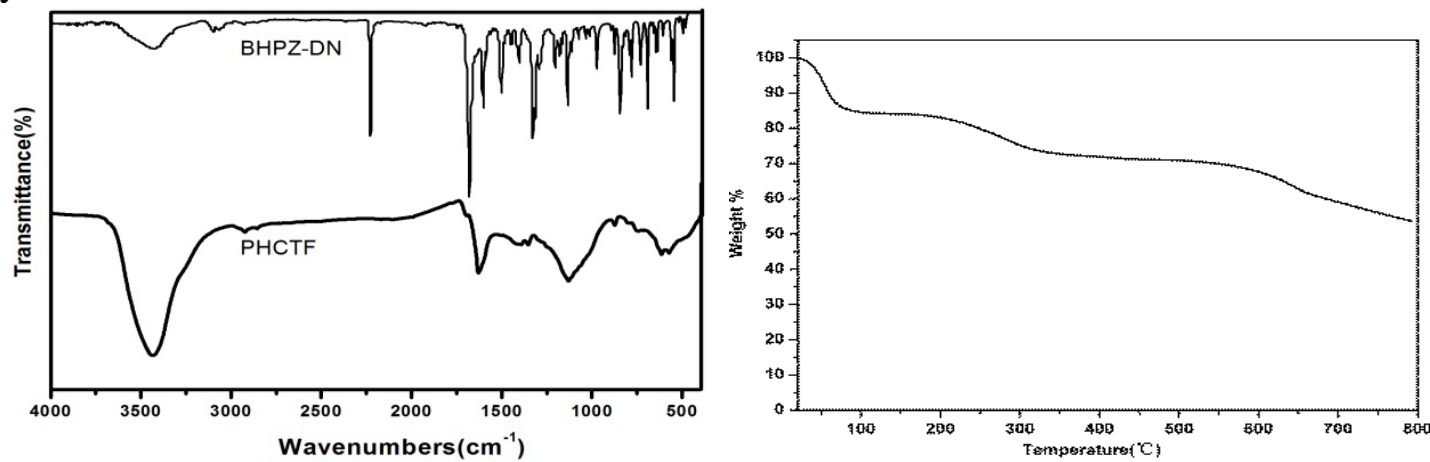

Fig 2. FT-IR spectra (Left) and thermogravimetric analysis plot of PHCTF (Right).

In order to characterize the nature of the pores of PHCTF, the porosities was evaluated by nitrogen sorption isotherm was recorded at $77 \mathrm{~K}$ from 0 to 760 Torr. Fig 3 (Left) shows the $\mathrm{N}_{2}$ adsorption/desorption isotherm of PHCTF. A sharp adsorption step can be noticed at a low relative pressure $\left(\mathrm{p} / \mathrm{p}_{0}\right)$ of 0 to 0.05 corresponding to gas sorption in micropores, which indicates the substantial microporous character (pore widths $<2 \mathrm{~nm}$ ). The adsorption isotherm follows a type- II character and the beginning of the almost linear middle section of the isotherm indicates the stage at which monolayer coverage is complete and multilayer adsorption takes place due to larger pores_ENREF_16 [13]. The desorption exhibits a slight H4-type hysteresis, that is, the adsorption and desorption branch of the isotherm remain nearly horizontal and parallel over a wide range of $\mathrm{p} / \mathrm{p}_{0}$. The apparent surface area calculated from the Brunauer-Emmett-Teller (BET) model over the pressure range of $\mathrm{p} / \mathrm{p}_{0}=0.05-0.1$ was $1062 \mathrm{~m}^{2} / \mathrm{g}$ as derived from nitrogen adsorption. Adopting the Langmuir model to the low-pressure region of the isotherm, the surface area was $1499 \mathrm{~m}^{2} / \mathrm{g}$. The pore size distribution (Fig 3 Right) was estimated from nonlocal density functional theory (NLDFT) using the model of carbon as an adsorbent indicates that a significant fraction of the pores surface still originates from micropores with a diameter less than $2 \AA$. For PHCTF the additional mesopores are broadly distrusted between 20 and $60 \AA$.
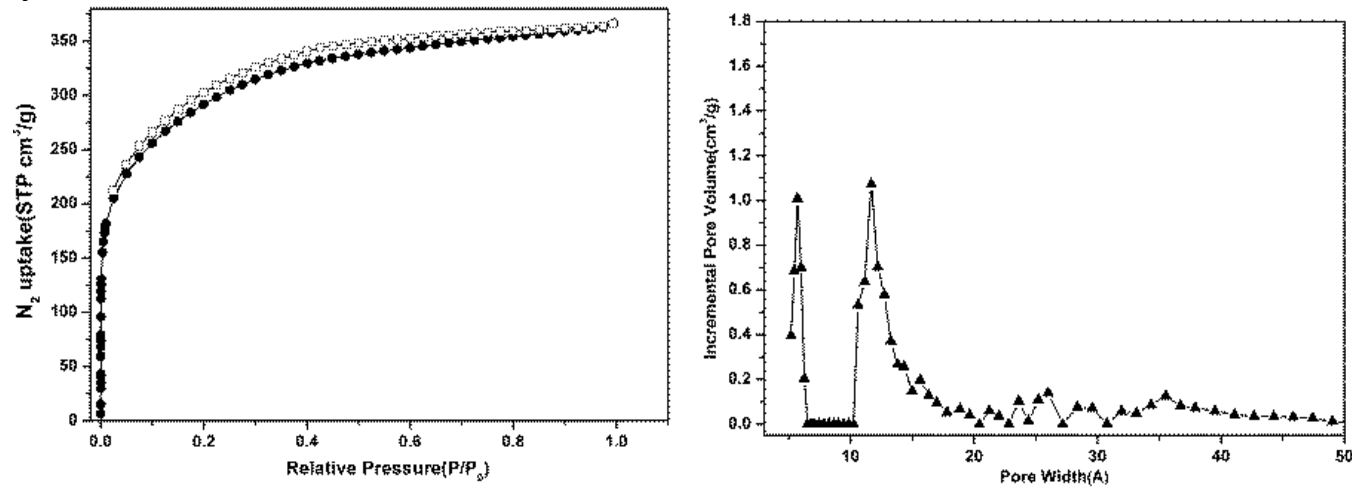

Fig 3. Nitrogen adsorption-desorption isotherm (a) and NLDFT pore size distribution curve (b)

\section{Conclusions}

A novel aromatic dinitrile with twisted phthalazinone moiety 2-(4'-cyanophenyl)-4-(4'cyanophenyl)-2,3-phthalazin-1-one (BHPZ-DN) was designed and synthesized as newly introduced building block, and a porous phthalazinone-based covalent triazine-based organic framework (PHCTF) was obtained at $400^{\circ} \mathrm{C}$ catalyzed by molten $\mathrm{ZnCl}_{2}$. FTIR spectra were used to confirm the formation of the trazine ring. The analysis of $\mathrm{N}_{2}$ sorption isotherm reveals that PHCTF exhibited substantial microporous and high apparent surface area. Simultaneously, TGA results showed that it had excellent heat resistance. 


\section{Acknowledgement}

The authors gratefully acknowledge the Fundamental Research Funds for the Central Universities of China (DUT13LK20) for its financial support of this research.

\section{References}

[1] Xu S, Luo Y, Tan B. Recent development of hypercrosslinked microporous organic polymers [J]. Macromolecular rapid communications. 2013,34(6):471-484.

[2] Du N, Park HB, Robertson GP, Dal-Cin MM, Visser T, Scoles L, et al. Polymer nanosieve membranes for CO2-capture applications [J]. Nat Mater. 2011,10(5):372-375.

[3] Liebl MR, Senker J. Microporous Functionalized Triazine-Based Polyimides with High CO2Capture Capacity [J]. Chemistry of Materials. 2013,25(6):970-980.

[4] Xiong S, Fu X, Xiang L, Yu G, Wang Z, Pan c, et al. Liquid acid-catalysed fabrication of nanoporous 1,3,5-triazine frameworks with efficient and selective CO2 uptake [J]. Polymer Chemistry. 2014.

[5] Tsyurupa MP, Davankov VA. Porous structure of hypercrosslinked polystyrene: State-of-the-art mini-review [J]. Reactive and Functional Polymers. 2006,66(7):768-779.

[6] Budd PM, Ghanem BS, Makhseed S, McKeown NB, Msayib KJ, Tattershall CE. Polymers of intrinsic microporosity (PIMs): robust, solution-processable, organic nanoporous materials [J]. Chemical communications. 2004(2):230-231.

[7] Cote AP, Benin AI, Ockwig NW, O'Keeffe M, Matzger AJ, Yaghi OM. Porous, crystalline, covalent organic frameworks [J]. Science. 2005,310(5751):1166-1170.

[8] Schmidt J, Werner M, Thomas A. Conjugated Microporous Polymer Networks via Yamamoto Polymerization [J]. Macromolecules. 2009,42(13):4426-4429.

[9] Kuhn P, Antonietti M, Thomas A. Porous, covalent triazine-based frameworks prepared by ionothermal synthesis [J]. Angewandte Chemie. 2008,47(18):3450-3453.

[10] Zhu X, Tian C, Mahurin SM, Chai SH, Wang C, Brown S, et al. A superacid-catalyzed synthesis of porous membranes based on triazine frameworks for CO2 separation [J]. Journal of the American Chemical Society. 2012,134(25):10478-10484.

[11] Bhunia A, Boldog I, Möller A, Janiak C. Highly stable nanoporous covalent triazine-based frameworks with an adamantane core for carbon dioxide sorption and separation [J]. Journal of Materials Chemistry A. 2013,1(47):14990-14999.

[12] Wu S, Liu Y, Yu G, Guan J, Pan C, Du Y, et al. Facile Preparation of Dibenzoheterocycle-Functional Nanoporous Polymeric Networks with High Gas Uptake Capacities [J]. Macromolecules. 2014:2875-2882.

[13] Matthias Thommes BS, Matthijs Groenewolt, Peter I. Ravikovitch and Alexander V. Neimark. Adsorption Hysteresis of Nitrogen and Argon in Pore Networks and Characterization of Novel Micro- and Mesoporous Silicas.pdf [J]. Langmuir. 2006,22:756-764. 